\title{
Prevalence of Medication Errors in Admitted Patients at the Philippine General Hospital
}

\author{
Paul Matthew D. Pasco, Ruzanne M. Caro, Connie L. Cruz, Nerissa M. Dando, Iris Thiele C. Isip-Tan, \\ Lynn R. Panganiban, Loralyn P. Pascua, Rosario R. Ricalde and Antonio C. Sison \\ UPCM Drug Safety Working Group
}

\begin{abstract}
Background. Medication errors are preventable events that can cause or lead to inappropriate drug use. Knowing the prevalence and types of errors can help us institute corrective measures and avoid adverse drug events.
\end{abstract}

Objective. This study determined the prevalence of medication errors and its specific types in the four main service wards of a tertiary government training medical center.

Methods. This is a retrospective, descriptive chart review study. From the master list of admissions, systematic sampling was done to retrieve the required number of charts. Relevant pages such as order sheets, nurses' notes, therapeutic sheets were photographed. For prolonged admissions, only the first 7 days were reviewed. Each chart was evaluated by two people who then met and agreed on the errors identified.

Results. The overall prevalence of medication errors is $97.8 \%$. Pediatrics had the most (63.3/chart), followed by Medicine, OBGynecology, and Surgery (7.3/chart). The most common type of errors identified were prescribing, followed by compliance, then administration errors.

Conclusion. Medication errors are present in the four main wards in our hospital. We recommend orientation of all incoming first year residents on proper ordering and prescribing of drugs, as well as a prospective observational study to determine true prevalence of all types of medication errors.

Key Words: medication error/s, drug use error/s, prescribing, dispensing, administration or compliance error/s

\section{Introduction}

The National Coordinating Council for Medication Error Reporting and Prevention of the UK defines

Presented at the 1st ASEAN Patient Safety Congress, June 26, 2015, Sofitel Philippine Plaza, Manila.

Corresponding author: Paul Matthew D. Pasco, MD, MSc

Department of Neurosciences

Philippine General Hospital

University of the Philippines Manila

Taft Avenue, Ermita, Manila, 1000 Philippines

Telefax No.: +6325548462

Email: pasco.paul@gmail.com medication errors as any preventable event that may cause or lead to inappropriate medication use or patient harm while the medication is in the control of the healthcare professional, patient or consumer. Medication error is failure to perform an intended action or performing a task that was not in the plan. ${ }^{1}$

There are five (5) types of medication errors according to stage of the medication process. Prescribing errors include incorrect drug selection, dose, dosage form, and illegible prescriptions. Dispensing errors include failure to dispense the correct drug in its right dose, dosage form, or dispensing the wrong drug. Administration errors include omission error, wrong time error, unauthorized drug error, improper dose error, wrong dosage-form error, wrong drug-preparation error, wrong administration-technique error, deteriorated drug error. Patient compliance errors involve monitoring and compliance errors. The last kind are potential errors which are detected and corrected through intervention before actual drug administration. ${ }^{1}$

Medication errors are common occurrences in clinical settings. In the local setting, the extent and scope of medication errors have not been fully established in the PGH since routine audit is limited to the intensive care units (ICU) and the $7^{\text {th }}$ pay floor ward. The Training, Research and Clinical Services Division of the Department of Pharmacy of the PGH began monitoring incidence of medication errors among patients in selected areas of the hospital (medicine, pediatrics, medical and central intensive care units and $7^{\text {th }}$ pay floor) from 2009 to 2011. A total of 3,287 observations were made, with an $8.4 \%$ prevalence rate of medication errors. In this report, the most common type of errors were transcription errors followed by prescribing errors. ${ }^{2}$

There is a growing concern with regard to medication errors since there has been an observable increase in the number of deaths from medication errors and adverse drug reactions in hospitals in the United States. Statistics showed that from 1983 to 1993, there was an increase from 2,876 cases to 7,391 cases. $^{3}$ In the United Kingdom, the same observation was noted in that the annual number of deaths due to medication errors increased from 20 to about 200 from 1990 to $2000 .{ }^{3}$ Medication errors can lead to adverse drug events, some of which are preventable or ameliorable. Hence the importance of avoiding medication errors. 


\section{Objective}

This study aimed to determine the frequency and specific types of medication errors in the four main service wards of PGH which are Medicine, Surgery, Pediatrics, and OB-Gynecology. Knowing the prevalence and types of errors can help hospital administration institute corrective measures and avoid adverse drug events.

\section{Methods}

This is a retrospective, descriptive chart review study of admitted patients from the four main service wards (Medicine, Pediatrics, Obstetrics-Gynecology and Surgery) of the Philippine General Hospital from January 1 December 31, 2013. The study was approved by the Research Ethics Board of the University of the Philippines Manila.

Using the formula of sample size for estimating a proportion, and using previous estimates of an $8.4 \%$ prevalence of medication errors in PGH, 120 charts per service, or a total of 480 charts, were needed to determine the medication error rate. From a master list of admissions provided by the Records Section, systematic sampling was done to retrieve the required number of charts. Only charts from charity ward patients were eligible to be included in the study. If the charts could not be located, the next patient on the list was considered. All relevant pages - order sheets, nurses' notes, and therapeutic sheets were photographed. For prolonged admissions, only the first 7 days were reviewed. An orientation and standardization workshop for all chart evaluators, who were also the study authors, was done at the start of the study. Each chart was evaluated separately by two individuals who then met and agreed on the errors identified. If there was no agreement between the two evaluators, they met and discussed the errors until a consensus was reached.

Data from the charts were pooled and no identifying data was collected. Since only retrospective data from the medical records were collected and data processing was anonymized, prospective consent taking was impracticable. The authors are all affiliated with the Philippine General Hospital.

\section{Results}

Of the 480 charts retrieved, only 460 were evaluated since some of the charts had incomplete data, or were private or ICU patients. Only 10 charts did not have a medication error, i.e. 450 out of 460 charts had at least one error with a $97.8 \%$ prevalence rate. Table 1 shows the frequencies of all types of medication errors per ward. Pediatrics had the most errors at 63.3 per chart, followed by Medicine, OBGynecology, and Surgery with the least at 7.3 per chart.

The most common type of errors identified in all departments were prescribing errors, followed by patient compliance errors, then administration errors (Figure 1). There were also unclassified errors, such as when a
Table 1. Frequencies of medication errors per department

\begin{tabular}{lccc}
\hline \multicolumn{1}{c}{ Department } & $\begin{array}{c}\text { No. of Medication } \\
\text { Errors }\end{array}$ & $\begin{array}{c}\text { No. of } \\
\text { Charts }\end{array}$ & $\begin{array}{c}\text { No. of errors per } \\
\text { chart (mean) }\end{array}$ \\
\hline Medicine & 3946 & 101 & 39.07 \\
Obstetrics- & 2409 & 119 & 20.24 \\
Gynecology & & & \\
Pediatrics & 7219 & 115 & 63.32 \\
Surgery & 872 & 115 & 7.27 \\
TOTAL & 14,446 & 450 & 32.10 \\
\hline
\end{tabular}

medication was not given but the reason, whether an omission of the nurses or lack of funds to buy the drug, was not clear.

Figure 2 shows an example of an illegible order, which is a prescribing error. Figure 3 shows the different types of prescribing errors; the short section in the figure shows a total of 14 different prescribing errors (incorrect abbreviation, no dose, no route, and no duration).

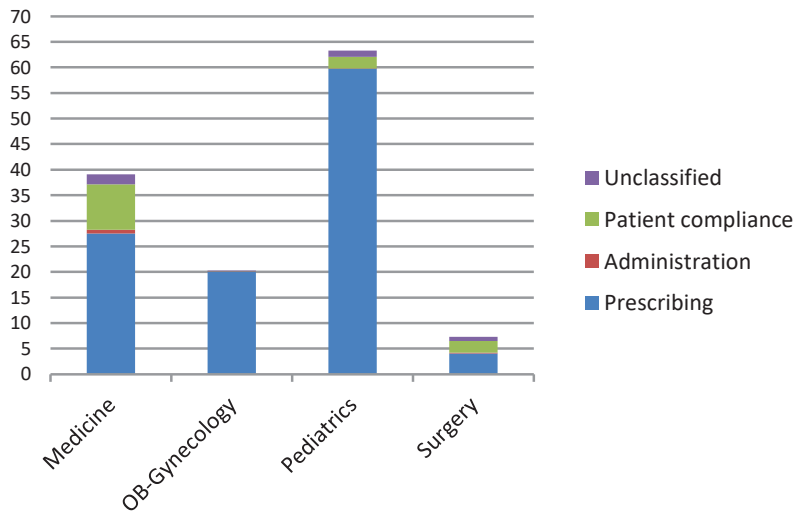

Figure 1. Number and types of medication errors per chart per department.

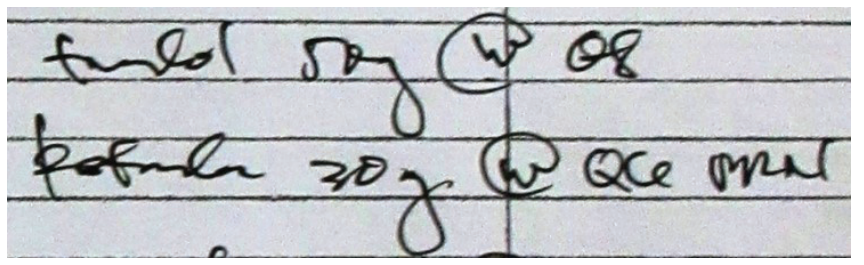

Figure 2. Example of an illegible order (prescribing error).

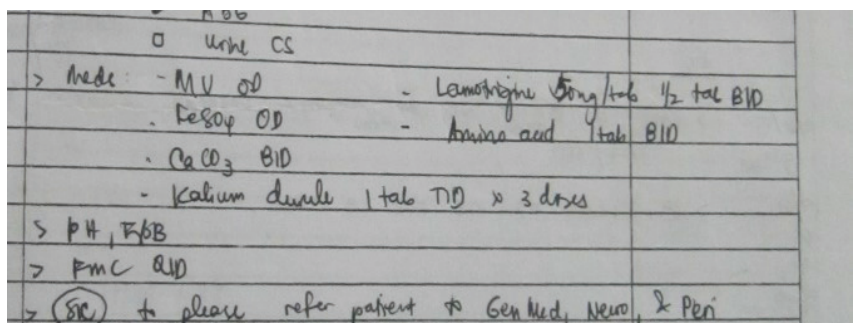

Figure 3. Example of different types of prescribing errors. The first line ("MV OD") has four different prescribing errors: incorrect abbreviation, no route, no dose, and no duration. 
The most common drugs involved in medication errors per department were: 1) Internal Medicine: omeprazole, lactulose, piperacillin-tazobactam, and aspirin; 2) OBGynecology: ferrous sulphate, multivitamins, celecoxib, and calcium carbonate; 3) Pediatrics: paracetamol, amikacin, ceftazidime, and salbutamol; and 4) Surgery: tramadol, cefuroxime, metronidazole, and omeprazole.

\section{Discussion}

Patient safety will always be a top priority in any health facility. With the growing concern in the increase in number of deaths from medication errors and adverse drugs reactions abroad, we are compelled to evaluate our operations. Since routine audit of medication errors is only done in the ICU (high risk areas) and certain pay floors, we wanted to extend this audit to other areas such as the four main service wards of the Philippine General Hospital. The $\mathrm{PGH}$ is manned mainly by trainees such as medical students, interns, residents, fellows, and student nurses and pharmacists. Although these trainees are supervised, the risk for having medication errors is certainly more apparent.

There were only 10 charts in the entire study which did not have a medication error. The prevalence rate of medication errors in the four main service wards thus is $97.8 \%$ which is significantly higher compared to the $8.4 \%$ reported in the ICU and pay floors in our hospital. The reason may be due to the more challenging clinical settings in these areas such as a high patient-to-doctor and patientto-nurse ratio and the limited amount of experience of these health personnel, who are typically medical residents in their first year of training. The discrepancy could also be due to differences in study methodology; our study being a retrospective chart review would easily pick up prescribing errors, whereas the study by the PGH pharmacy department focused on administration and patient compliance errors.

Prescribing errors were found to be far more common compared to the other types of errors. This may be due to the nature of this study, which was purely a retrospective chart review. Hence errors in ordering and prescribing could be more easily picked up than administration and patient compliance errors, which depend on direct observation and monitoring of ward activities, as well as admission of errors on the part of nurses and other health care personnel.

The Surgery service ward had the least medication errors since they prescribed far fewer medications compared to Medicine and Pediatrics. Most of the medications prescribed were part of post-anesthesia care. The most common type of error was still prescribing error. The route was not specified and assumed to be per orem whenever the drug was in capsule or tablet form. The brand names were written instead of the generic names of the drugs with more than one component such as Tramadol + Paracetamol.
Noticeably, compliance errors were few since most of the cases in the Surgery ward were elective cases and the patients were given a list of medications to be used during confinement.

Depending on definition, prevalence of medication errors ranges from 1.2 - 140\%. The prevalence rate determined in this study is within this range, on the higher end. In the study done by the Committee on Identifying and Preventing Medication Errors by the Institute of Medicine in 2007, the incidence rates of medication errors in the hospital settings would vary from one hospital to another. There was an average of 9 error types identified in 38 of the 45 studies and dosing errors were the most frequent single error type. In England, in a study involving 124,260 prescriptions in 19 hospitals in North-West England over a 7-day period, the over-all rate of medication error was $8.9 \%$ and $1.7 \%$ of errors were potentially lethal. The top 5 drugs were analgesics (9.7\%), antibacterial drugs $(6.2 \%)$, bronchodilators $(5.7 \%)$, anti-anginal drugs $(5.3 \%)$ and corticosteroids $(5.9 \%)$. The types of errors were omission on admission to hospital $(29.8 \%)$, underdosage $(11.1 \%)$, overdosage $(8.5 \%)$ and significant allergy $(0.3 \%){ }^{1}$

Kaushal, et al. (2001) performed a study on medication errors and adverse drug events in pediatric in-patients in 2 academic institutions in the U.S. over a 6-week period. Of 10,778 medication orders, 616 (5.7\%) were medication errors, $115(1.1 \%)$ were potential adverse drug events (ADEs) and $26(0.24 \%)$ were ADEs. Of the 26 ADEs, 5 or $19 \%$ were preventable. The rate of potential ADEs increased in neonates in the neonatal intensive care unit. The common types of medication errors were again prescribing errors $(79 \%)$, administrative errors (34\%). The drugs involved were anti-infective medications $(28 \%)$ and intravenous drugs $(54 \%){ }^{5}$

In 1993-1998, the US-FDA study showed that the most common medication error was related to administration of an improper dose of medicine, accounting for $41 \%$ of fatal medication errors. The administration of the wrong drug and using the wrong route of administration each accounted for $16 \%$ of medication errors. Almost half of the fatal medication errors occurred in people over the age of 60. Older people may be at greater risk for medication errors because they often take multiple medications. ${ }^{6}$ A study by Barker KN, et al. (2002) done in the U.S, involving 36 institutions showed that $19 \%$ of the doses (605/3216) were erroneous. The most frequent errors by category were wrong time (43\%), omission (30\%), wrong dose $(17 \%)$, and unauthorized drug $(4 \%)$. Seven percent of the errors were judged potential adverse drug events. The study concluded that medication errors were common, occurring in 1 of every 5 doses in a typical hospital and skilled nursing facility. The percentage of errors that rated to be potentially harmful was $7 \%$, or more than 40 per day in a typical 300-patient institution. ${ }^{7}$ 
There are also several types of methods to detect errors, each with their own advantages and disadvantages. ${ }^{8}$ Methods other than chart review, such as incident reporting, voluntary reporting, and direct care observation may perhaps be used in order to increase detection of the other types of medication errors that were not captured in this study.

In 2012, the Institute for Safe Medication Practices in the United States updated the list of "high-alert" drugs that potentially can cause harm to admitted patients when erroneously used..$^{9}$ Majority in the list are being used in the $\mathrm{PGH}$, such as adrenergic agonists (epinephrine, norepinephrine), adrenergic antagonists (metoprolol), anesthetics (propofol), antiarrhythmics (lidocaine), antithrombotics (anticoagulants and fibrinolytics), anti-diabetic drugs (oral hypoglycemic, insulin), narcotics/opioids and chemotherapeutic agents.

Finally, the most common drugs involved in medication errors per department were: omeprazole for Internal Medicine, ferrous sulfate for OB-Gynecology; paracetamol for Pediatrics, and tramadol for Surgery. This may simply be due to the fact that these are among the most common drugs prescribed in each department. Note that none of these are among the "high-alert" drugs which require extra precaution as they may cause serious patient harm when used in error, but they still have the potential for adverse drug reactions.

In this study, prescribing errors were the most common type of medication errors detected. To achieve balanced prescribing, Aronson ${ }^{10}$ recommended that the prescriber think through questions on indication, effectiveness, interactions, dosage, and economics, among others, before writing a prescription.

\section{Conclusion and Recommendations}

There is a high prevalence of medication errors in the main service wards at the Philippine General Hospital at 97.8\% prevalence rate with Pediatrics and Medicine having the most errors. The most common type of medication error is prescribing. Since residents-in-training are the ones prescribing in these areas, we recommend orientation of residents on proper ordering and prescribing of drugs. Also, a prospective in-situ observational study should be performed in order to obtain a more accurate prevalence figure of the various types of medication errors.

Statement of Authorship

All authors have approved the final version submitted.

\section{Author Disclosure}

All the authors declared no conflicts of interest.

\section{Funding Source}

This paper was funded by the University of the Philippines College of Medicine.

\section{References}

1. Lisby M, Nielsen LP, Brock B, Mainz J. How are medication errors defined? A systematic literature review of definitions and characteristics. Int J Qual Health Care. 2010; 22(6):507-18.

2. Department of Pharmacy, UP-Philippine General Hospital. Summary of Pharmacists' Intervention, 2009-2011.

3. Aronson JK. Medication errors: what they are, how they happen, and how to avoid them. QJM. 2009; 102(8):513-21.

4. Glavin RJ. Drug errors: consequences, mechanisms, and avoidance. Br J Anaesth. 2010; 105(1):76-82.

5. Kaushal R, Bates DW, Landrigan C, et al. Medication errors and adverse drug events in pediatric inpatients. JAMA. 2001; 285(16):2114-20.

6. Aspden P, et al. (eds). Preventing Medication Errors: Quality Chasm Series. National Academy of Sciences; 2007. pp. 1-447.

7. Barker KN, Flynn EA, Pepper GA, Bates DW, Mikeal RL. Medication errors observed in 36 health care facilities. Arch Intern Med. 2002; 162(16):1897-903.

8. Montesi G, Lechi A. Prevention of medication errors, detection and audit. Br J Clin Pharmacol. 2009; 67(6):651-5.

9. Institute for Safe Medication Practices. List of High Alert Medications [Online]. [cited 2015 July]. Available from https://www.ismp.org/ tools/highalertmedications.pdf.

10. Aronson JK. Medication errors: EMERGing solutions. Br J Clin Pharmacol. 2009; 67(6):589-91. 